\title{
Deutschunterricht in Firmenkursen II Ergebnisse der Umfrage vom Sommer 2009
}

\section{Pavla Marečková}

In the last few years, the number of companies and institutions that organize a language course for their employees has been growing rapidly. From the viewpoint of methodology, however, there is a lack of technical literature discussing the regularities of such language courses. That is why a survey was conducted with teachers and participants of these courses that was meant to outline the present situation. The paper at hand presents the evaluation of the students' answers. The results including the teachers' responses are available within the last issue of this magazine (2009/2).

company language courses - German as a foreign language - foreign language teaching language polls - questionnaire

Die Zahl der Firmen bzw. Institutionen, die für ihre Angestellten einen Sprachkurs organisieren, nimmt in den letzten Jahren stark zu. Aus didaktischer Sicht fehlt es jedoch an Fachliteratur, die die Gesetzmäßigkeiten eines solchen Fremdsprachenunterrichts komplex behandeln würde. Daher wurde eine Umfrage unter LektorInnen und KursteilnehmerInnen durchgeführt, die eine Vorstellung über die gegenwärtige Situation ermöglichen sollte. Im vorliegenden Beitrag werden die Ergebnisse präsentiert, die die Antworten der KursteilnehmerInnen widerspiegeln. Die Ergebnisse der Umfrage, die unter LektorInnen durchgeführt wurde, wurden in der vorigen Nummer dieser Zeitschrift (2009/2) veröffentlicht.

Firmenunterricht - Firmenkurs - Umfrage - Deutsch als Fremdsprache Fremdsprachendidaktik

\section{Einleitung}

Die Zahl der Firmen bzw. Institutionen, die für ihre Angestellten einen Sprachkurs organisieren, nimmt in den letzten Jahren stark zu. Dieses Interesse an leb en slangem Fremdsprachenlernen für den Beruf wird auch von der Sprachenpolitik der Europäischen Union unterstützt (GERR: 2000). Obwohl heutzutage Englisch als „Lingua Franca“ betrachtet wird, wird Deutsch für Erwachsene im Vergleich mit dem Englischen in Tschechien etwa gleich nachgefragt (NEKULA: 2004, 129). Von dieser Seite dürften auch neue Impulse für das tschechische Bildungssystem ausgehen.

In der vorigen Ausgabe der Brünner Hefte (2009/2) wurde im Rahmen des Artikels Deutschunterricht in Firmenkursen I (MAREČKOVÁ: 2009) der erste Teil der Ergebnisse veröffentlicht, die einer Umfrage vom Mai bis August 2009 
entnommen wurden. ${ }^{1}$ Das Ziel der Umfrage war, die Hauptmerkmale des heutigen Deutschunterrichts in Firmenkursen zu beschreiben und somit aus Sicht der Fremdsprachendidaktik einen Einblick in die Problematik zu gewinnen. Konkret haben wir uns die Frage gestellt, wie die Firmenkurse organisatorisch abgesichert werden, worin die Motivation der KursteilnehmerInnen zum Belegen eines Sprachkurses besteht und nicht zuletzt welche methodisch-didaktischen Gesetzmäßigkeiten sich in den Firmenkursen feststellen lassen.

$\mathrm{Zu}$ diesem Zweck wurden sowohl LektorInnen als auch KursteilnehmerInnen verschiedener Firmenkursen in Brünn bzw. in der Umgebung ${ }^{2}$ online befragt. Die Ergebnisse der Umfrage unter den LektorInnen wurden, wie schon erwähnt, in der vorigen Nummer dieser Zeitschrift präsentiert. In diesem Beitrag gehen wir also direkt auf die Antworten der KursteilnehmerInnen ein.

\section{Der Fragebogen}

Der für die KursteilnehmerInnen bestimmte Fragebogen bestand aus 16 Items. Die elektronische Form des Fragebogens wurde an ca. 80 KursteilnehmerInnen per E-Mail verschickt, von denen 44 ein komplett ausgefülltes Formular zurückschickten. Die meisten Items wurden als geschlossene bzw. halb-offene Fragen formuliert. Am Ende des Fragebogens wurden die KursteilnehmerInnen aufgefordert, eigene Erfahrungen oder Anregungen bezüglich des Fremdsprachenunterrichts in Firmenkursen zu formulieren.

\section{Datenauswertung und Ergebnisse}

Für die Auswertung der erfassten Daten wurde die Software Statistica 9 verwendet. Die Ergebnisse werden in Form von Säulen- bzw. Tortendiagrammen oder Tabellen dargestellt und beziehen sich nur auf diese Stichprobe (44 ProbandInnen). Da die Stichprobe nicht genügend groß ist und dadurch die Prozentsätze verzerrend wirken könnten, fügen wir in den jeweiligen Graphiken neben den Prozentangaben auch die Anzahl der Antworten hinzu.

Frage Nr. 1: Wann findet der Sprachkurs statt?

1 Die Untersuchung konnte dank finanzieller Unterstützung des Forschungsprojektes MUNI/41/006/2009 der Masaryk-Universität durchgeführt werden. Das Projekt bzw. die dadurch gewonnenen Daten dienten als Basis für die Erstellung eines neuen Fortbildungskurses für DeutschlehrerInnen, die sich auf den Firmenunterricht spezialisieren. Der Kurs wird seit Herbst 2010 am Lehrstuhl für deutsche Sprache und Literatur der Pädagogischen Fakultät der MasarykUniversität angeboten.

2 Die Firmenkurse wurden auf Grund persönlicher Kontakte ausgewählt. Die Wirtschaftszweige, in denen die jeweiligen Firmen tätig sind, wurden bei der Auswahl nicht berücksichtigt. 
Durch die erste Frage sollte festgestellt werden, ob der Sprachunterricht innerhalb oder außerhalb der Arbeitszeit stattfindet.

\section{Wann findet der Sprachkurs statt?}

außerhalb der Arbeitszeit; 13; 30\%

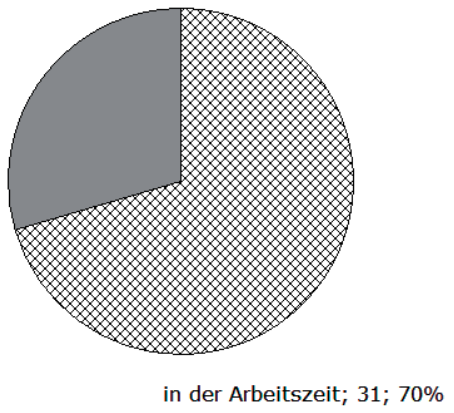

Auf den ersten Blick ist der Graphik zu entnehmen, dass in $70 \%$ der Fälle der Sprachunterricht im Rahmen der Arbeitszeit abgehalten wird. Die restlichen $30 \%$ der untersuchten Sprachkurse finden vor oder nach der Arbeitszeit statt.

Frage Nr. 2: Wie oft findet der Sprachkurs statt?

Die Frequenz des Sprachunterrichts gehört zu den Schlüsselfaktoren, die den Lernfortschritt beeinflussen. Optimal scheinen zwei Doppelstunden in der Woche zu sein. Nichtsdestoweniger sieht die Realität oft anders aus, was auch unsere Ergebnisse bestätigen.

\section{Wie oft findet der Sprachkurs statt?}

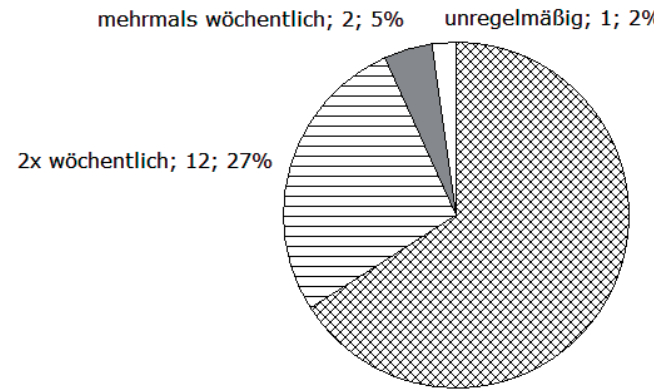

1x wöchentlich; 29; $66 \%$ 
Ungefährin einem Drittel der untersuchten Kursetreffen sich dieKursteilnehmerInnen zum Sprachunterricht zweimal oder sogar mehrmals pro Woche. Was jedoch stark überwiegt, sind die Sprachkurse, die nur einmal pro Woche stattfinden (66\%). Einer der Kurse wird sogar nur unregelmäßig abgehalten.

Frage Nr. 3: Wo findet der Sprachkurs statt?

Das Ziel dieser Frage war festzustellen, in welchen Räumlichkeiten der Sprachunterricht gewöhnlich stattfindet (besonders dann, wenn der Unterricht nicht an einer Sprachschule abgehalten wird).

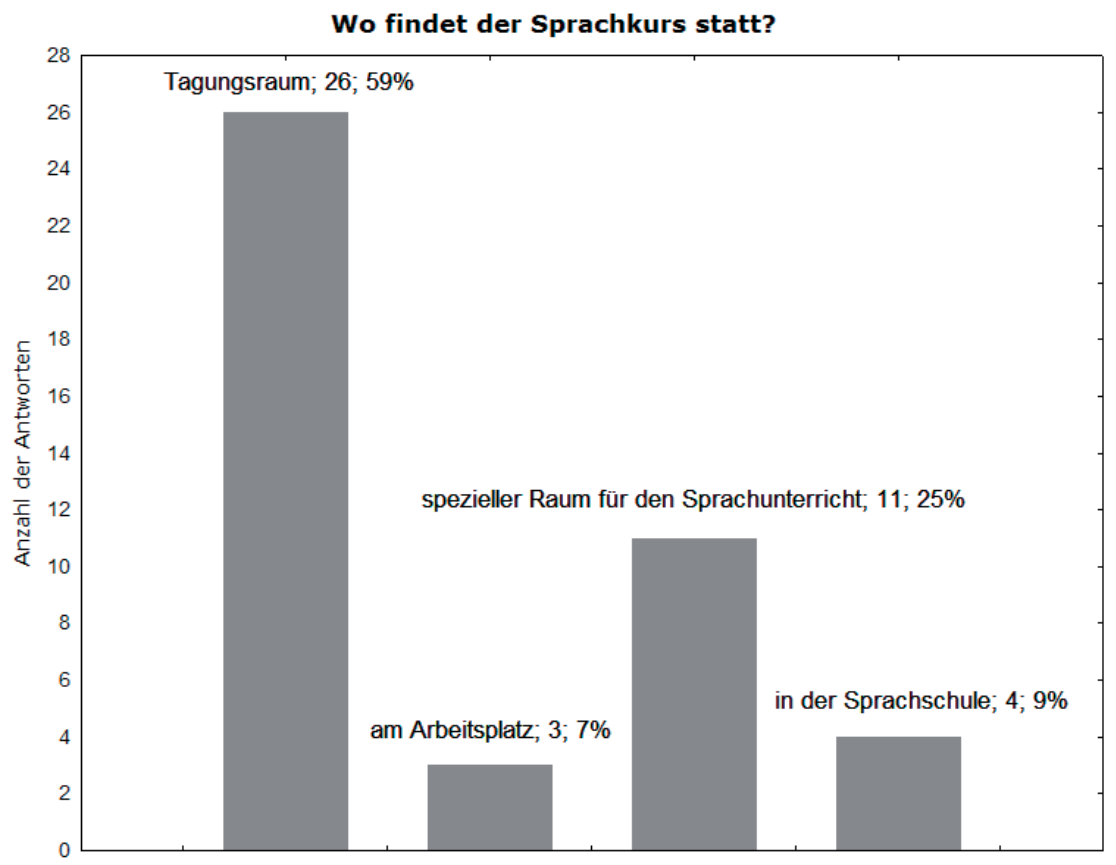

Aus der Umfrage geht hervor, dass die meisten untersuchten Kurse direkt in den Räumlichkeiten der jeweiligen Firma stattfinden. Die RespondentInnen gaben am häufigsten an, dass der Tagungsraum für den Sprachkurs vorgesehen ist (59 \%). Laut den Aussagen gibt es in manchen Firmen auch spezielle Räume für den Sprachunterricht (25\%). In drei Fällen lernen die KursteilnehmerInnen an ihrem eigenen Arbeitsplatz (7\%), was höchstwahrscheinlich besonders die Einzelkurse betrifft. 
Frage Nr. 4 Wer trägt die Kosten für den Sprachunterricht?

Das nächste Item im Fragebogen betraf einen nicht weniger wichtigen Faktor des Fremdsprachenlernens, und zwar die Kosten für den Sprachkurs.

\section{Wer trägt die Kosten für den Sprachunterricht?}

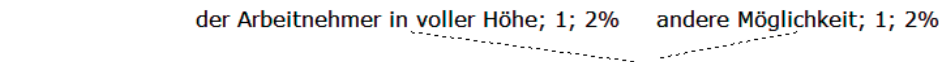

der Arbeitgeber und -nehmer teilen die Kosten; 3; $7 \%$

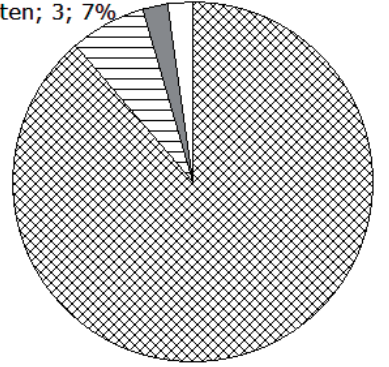

der Arbeitgeber in voller Höhe; $39 ; 89 \%$

Wie die Graphik verdeutlicht, ist es meistens der/die ArbeitgeberIn, der/die die Kosten für den Sprachkurs in voller Höhe übernimmt (89 \%). In drei Fällen werden die Kosten geteilt und in einem Fall bezahlt der/die ArbeitnehmerIn die Kursgebühr selbst. Interessant ist besonders eine Antwort, der zufolge die Kosten nur dann von der/dem ArbeitgeberIn gedeckt werden, wenn der/die ArbeitnehmerIn gewisse Kriterien erfüllt, wie z. B. Anwesenheit im Unterricht oder eine bestimmte Punktezahl beim Abschlusstest.

Frage Nr. 5 Aus welchem Grund besuchen Sie den Sprachkurs?

Durch diese Frage wollten wir erfahren, ob die KursteilnehmerInnen den Sprachkurs besuchen, weil dies die Firmenleitung fordert, oder eher aus eigener Überzeugung. 


\section{Aus welchem Grund besuchen Sie den Sprachkurs?}

andere Gründe; $1 ; 2 \% \quad$ pflichtige Teilnahme; $12 ; 27 \%$

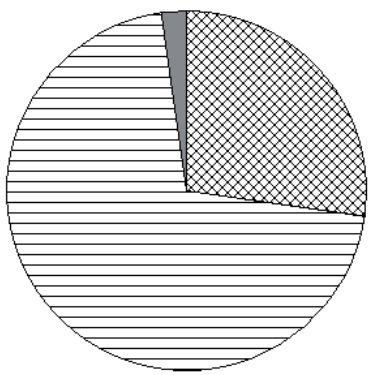

freiwillige Teilnahme; 31; 70\%

Mehr als zwei Drittel der befragten KursteilnehmerInnen besuchen den Sprachkurs freiwillig, $27 \%$ der RespondentInnen gaben hingegen an, dass die Teilnahme am Fremdsprachunterricht von der Firmenleitung verlangt wird. In einem Fall wurde als Grund für die Teilnahme eine Kombination der beiden oben genannten Möglichkeiten genannt. Einerseits kann es als erfreulich gelten, dass die KursteilnehmerInnen den Sprachunterricht aus eigener Überzeugung besuchen, andererseits bedeutet dies nicht automatisch, dass sie dadurch auch besser motiviert sind. Auf die Problematik der Motivation geht die nächste Frage ein:

Frage Nr. 6 Worin besteht ihre Motivation einen Sprachkurs zu belegen?

Bei dieser Frage konnten die KursteilnehmerInnen mehrere Antworten ankreuzen. Zur Auswahl bekamen Sie folgende Alternativen: Ich muss/soll mit ausländischen GeschäftspartnerInnen kommunizieren; Ich will meine Fremdsprachenkenntnisse erweitern (bzw. das bisherige Sprachniveau halten); Ich will mich weiterbilden; Ich will eine Sprachprü̈ungablegen (internationales Zertifikat, Sprachdiplom, etc.); die Teilnahme an dem Sprachkurs ist für mich günstig (die Kosten trägt der/die ArbeitgeberIn). 


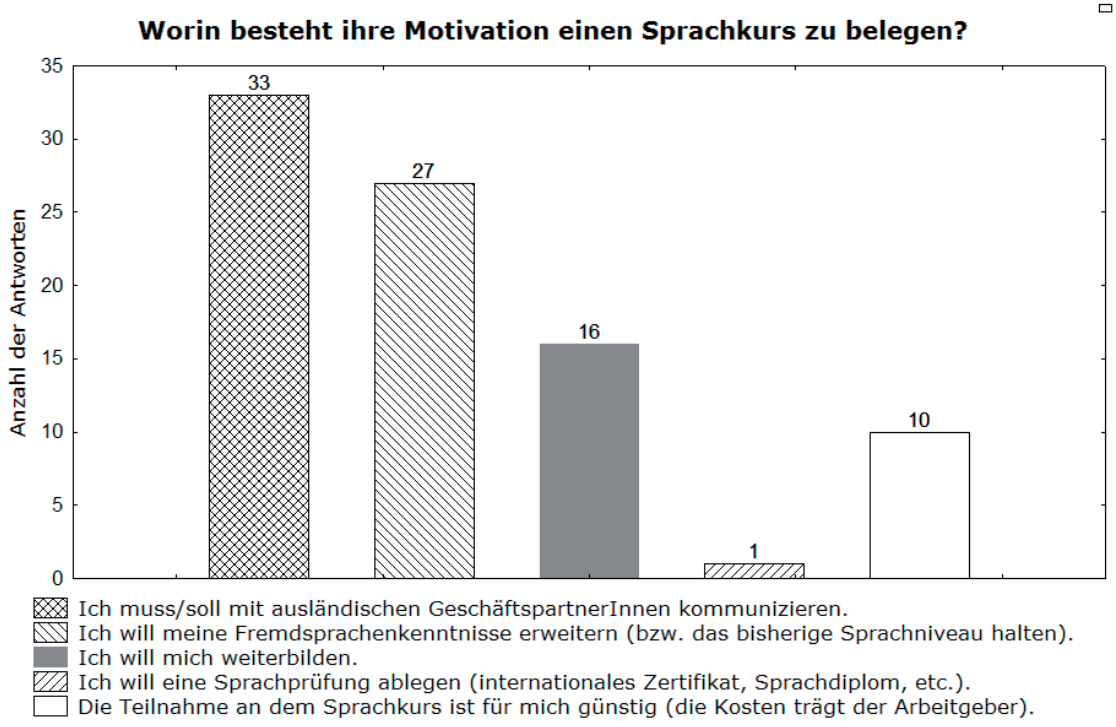

$\mathrm{Da}$ in diesem Fall mehrere Antworten markiert werden konnten, sind in der Graphik nur absolute Zahlen ohne Prozentangaben angeführt. Wie die Graphik illustriert, wurde die erste Möglichkeit am häufigsten markiert (33x), was heißt, dass diese RespondentInnen die Fremdsprache für ihren Beruf brauchen. Den nächsten, auch relativ starken, Motivationsfaktor stellt das Bedürfnis dar, die eigenen Sprachkenntnisse zu vertiefen (27x). Aus der Grafik geht weiterhin hervor, dass die KursteilnehmerInnen den Sprachkurs auch deshalb besuchen, weil sie sich weiterbilden wollen (16x) oder weil es für sie finanziell vorteilhaft ist (10x). Die Ablegung einer Sprachprüfung wurde nur einmal als Motivationsfaktor angegeben.

\section{Frage Nr. 7 Was für einen Sprachkurs besuchen Sie?}

Heutzutage gibt es verschiedene Typen von Sprachkursen, die angeboten werden. Was den Firmenunterricht anbelangt, haben wir es am häufigsten mit Gruppenkursen oder Kursen für einzelne Personen, d. h. Individualkursen, zu tun. Zwei Drittel der befragten KursteilnehmerInnen (67\%) besuchen einen Gruppen-Sprachkurs und ein Drittel (33\%) wird individuell unterrichtet. Da nur zwei der Antwortmöglichkeiten gewählt wurden, ist eine graphische Darstellung überflüssig.

Frage Nr. 8 Falls Sie einen Gruppen-Sprachkurs besuchen, wie läuft der Unterricht meistens ab? 
Ähnlich wie bei Frage Nr. 6 konnten die RespondentInnen auch hier mehrere Antworten ankreuzen. Das Ziel dabei war festzustellen, welche Sozialform am häufigsten verwendet wird. Zur Auswahl standen folgende Alternativen: Frontalunterricht, Gruppenarbeit, Partnerarbeit, Einzelarbeit, alle oben genannten Sozialformen werden miteinander kombiniert oder andere Sozialform. Der letztgenannten Antwort konnte eine Beschreibung beigefügt werden.

Falls Sie einen Gruppen-Sprachkurs besuchen, wie läuft der Unterricht meistens ab?

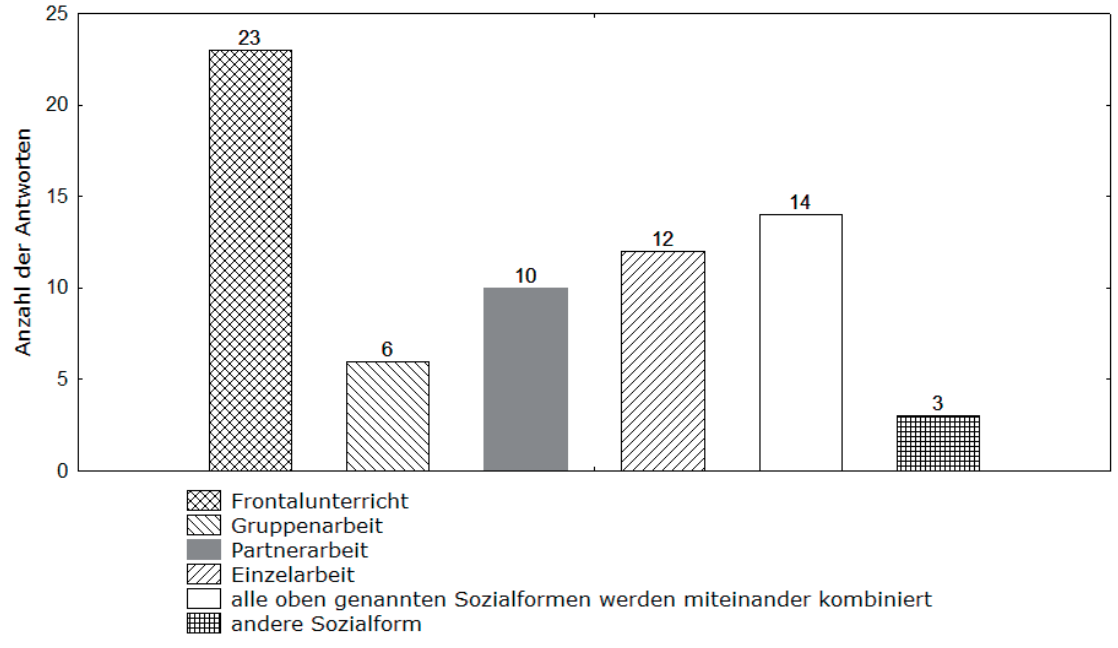

Laut der Umfrage überwiegt der Frontalunterricht (23x). Außerdem arbeiten die KursteilnehmerInnen auch selbständig (12x) oder in Paaren (10x). In Gruppen wird nur selten gearbeitet $(6 \mathrm{x})$. Nicht zuletzt wurde angegeben, dass die verschiedenen Sozialformen miteinander kombiniert werden, was allerdings nur ein Drittel der RespondentInnen angab. In drei Fällen wurde zwar eine andere Sozialform angegeben, die Antworten entsprechen jedoch nicht der Fragestellung (Wir reden miteinander oder Konversation).

Frage Nr. 9 Welche Unterrichtsmaterialien werden im Sprachkurs verwendet?

DieAuswahlvonUnterrichtsmaterialienbleibtmeistensdem/derLektor/inüberlassen. Als Erstes muss er/sie, vielleicht auch zusammen mit den KursteilnehmerInnen, die Wahl treffen, ob mit einem Lehrwerk gearbeitet wird oder lieber mit Texten und Übungen, die der/die Lektor/in dem Lernziel gemäß zusammenstellt und für alle kopiert. Beide Möglichkeiten haben ihre Vor- und Nachteile, daher wird oft 
eine Kompromisslösung gewählt: Das Lehrwerk, das die Struktur des Sprachkurses bestimmt, wird durch andere Unterrichtsmaterialien angereichert.

\section{Welche Unterrichtsmaterialien werden im Sprachkurs verwendet?}

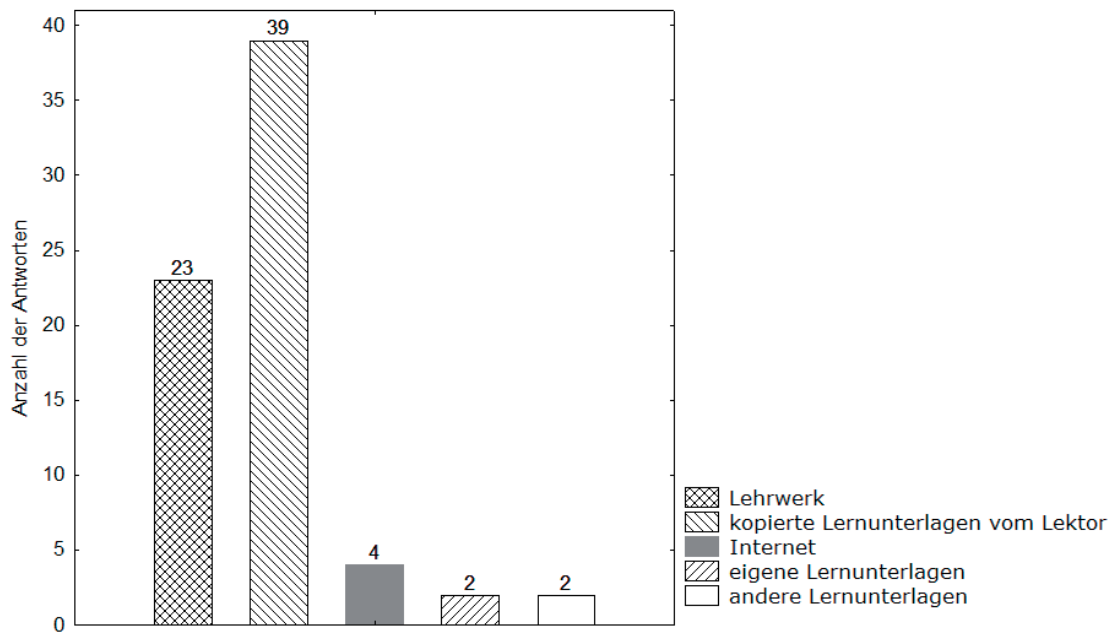

Bei dieser Frage konnten die KursteilnehmerInnen wieder mehrere Antworten ankreuzen. Die Graphik zeigt, dass die Hälfte der Befragten mit einem Lehrwerk im Sprachkurs arbeitet (z. B. Alltag und Beruf, Némecky súsmèvem (,Deutsch mit einem Lächeln') oder mit speziellen Berlitz-Lehrwerken. Nichtsdestoweniger bekommt die Mehrheit der Befragten kopierte Unterlagen im Unterricht (39x), was darauf hindeutet, dass der Firmenunterricht sehr spezifisch ist und man kaum mit nur einem Unterrichtsmaterial auskommt. Sehr selten werden auch eigene Lernunterlagen $(2 \mathrm{x})$ oder das Internet (4x) genutzt.

Frage Nr. 10 Wie viel Aufmerksamkeit wird folgenden Tätigkeiten gewidmet?

Unter der Bezeichnung „Tätigkeiten“ verstehen wir hier das Training einzelner Sprachfertigkeiten und Teilkompetenzen. Einfachheitshalber verzichteten wir jedoch im Fragebogen auf diese fachwissenschaftlichen Termini. Die KursteilnehmerInnen sollten jeder Fertigkeit bzw. Teilkompetenz eine Zahl auf einer Skala von 1 bis 5 zuordnen, je nachdem, ob sie sich der jeweiligen Tätigkeit sehr oft im Unterricht widmen (1) oder gar nicht widmen (5), wobei man die Eins höchstens zwei Mal verwenden durfte. Die Ergebnisse sind in der folgenden Tabelle nach dem Durchschnittswert geordnet. 
wird sehr oft gewidmet 1-2-3-4-5 wird gar nicht gewidmet

\begin{tabular}{|l|l|l|}
\hline $\begin{array}{l}\text { Platz in der } \\
\text { Reihenfolge }\end{array}$ & Tätigkeit & Durchschnittswert \\
\hline 1 & Konversation & 1,6 \\
\hline 2 & Wortschatz & 2,2 \\
\hline 2 & Grammatik & 2,2 \\
\hline 3 & Lesen & 2,4 \\
\hline 4 & Aussprache & 3,0 \\
\hline 5 & Hören & 3,1 \\
\hline 6 & Rechtschreibung & 3,5 \\
\hline 7 & Schreiben & 3,8 \\
\hline
\end{tabular}

An den Durchschnittswerten ist abzulesen, dass Konversationsübungen, Wortschatzarbeit und Grammatiktraining zu den häufigsten Aktivitäten in den untersuchten Firmenkursen gehören. Hingegen werden Schreibaufgaben bzw. Rechtschreibübungen nicht so oft gemacht. Diese Ergebnisse stimmen mit den Ergebnissen der Umfrage, die unter LektorInnen gemacht wurde (MAREČKOVÁ: 2009), überein.

\section{Frage Nr. 11 Wer leitet den Sprachkurs?}

Das Ziel dieser Frage war festzustellen, ob in den Firmenkursen MuttersprachlerInnen oder eher tschechische LektorInnen unterrichten. Aus den Antworten geht hervor, dass ungefähr $90 \%$ der Kurse von einem/einer tschechischen LektorIn und nur ca. $10 \%$ der Kurse von MuttersprachlerInnen geleitet werden. Im Rahmen dieser Frage sollten die ProbandInnen außerdem angeben, wen sie bevorzugen. Fast $60 \%$ wäre für einen/eine Tschechisch sprechende/n LektorIn.

Frage Nr. 12 und 13 waren auf die Bewertung der Kompetenzen von LektorInnen und der Atmosphäre im Sprachkurs gerichtet. Die meisten befragten KursteilnehmerInnen gaben an, dass sie mit ihren LektorInnen zufrieden sind und sie für kompetent halten. Eine ähnlich positive Bewertung ergab sich aus den Antworten auf die zweite Frage, denn 98 \% der RespondentInnen bewerten die Atmosphäre im Sprachkurs als angenehm bzw. eher angenehm.

Frage Nr. 14 Was stört den Unterricht im Firmenkurs? 
Bei dieser Frage wurden sowohl Alternativantworten als auch die Möglichkeit, eigene Aussage zu formulieren, zur Verfügung gestellt. Man konnte wieder mehrere Antworten markieren.

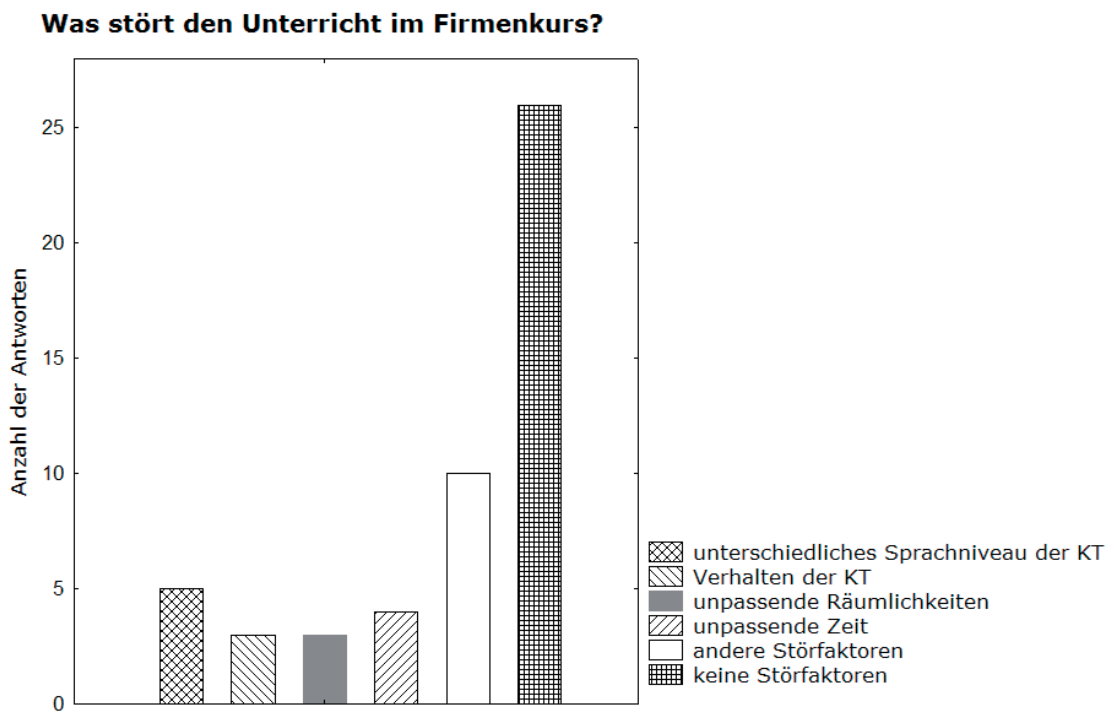

Laut den Ergebnissen scheint mehr als die Hälfte der KursteilnehmerInnen im Sprachkurs von nichts gestört zu werden. Im Vergleich dazu wurden die anderen Optionen deutlich seltener-markiert (unterschiedliches Sprachniveau 5x, Verhalten der KursteilnehmerInnen 3x, unpassende Räumlichkeiten 3x, unpassende Zeit 4x). In 10 Fällen wurden andere Störfaktoren angegeben, wie z. B. Überforderung in der Arbeit, unregelmäßige Präsenz, dienstliche Mobiltelefone, die eingeschaltet bleiben müssen, andere Prioritäten oder die Tatsache, dass man unvorbereitet zum Unterricht kommt.

Frage Nr. 15 Hat der Sprachkurs Ihre Erwartungen erfüllt?

Ähnlich wie bei Frage Nr. 12 oder 13 erschienen auch hier überwiegend positive Bewertungen. $91 \%$ der befragten KursteilnehmerInnen sind mit dem Sprachkurs völlig zufrieden (34\%) oder eher zufrieden (57\%). Die restlichen ProbandInnen (9\%) gaben an, dass sie mit dem Kurs eher unzufrieden sind.

Am Ende des Fragebogens wurden die KursteilnehmerInnen aufgefordert, weitere Anregungen bzw. Tipps bezüglich der Effizienz des Firmenunterrichts aufzuschreiben. 
Aus den Aussagen geht hervor, dass der Unterricht nur dann gut funktionieren kann, wenn der Kurs sozusagen maßgeschneidert ist. Das heißt, dass sowohl Inhalte als auch Sozialformen und Gestaltung der Unterrichtseinheit den Anforderungen der jeweiligen Lerngruppe angepasst werden müssen.

Aus Platzgründen wurden in diesem Artikel die Ergebnisse zu jeder Frage zusammenfassend präsentiert bzw. nur kurz interpretiert. Allerdings steht potenziellen Interessenten die vollständige Datenbank bei der Autorin des Artikels zur Verfügung. ${ }^{1}$

\section{Zusammenfassung}

Die Antworten auf die ersten vier Fragen zeigen, welchen Stellenwert der Sprachunterricht in den jeweiligen Firmen einnimmt. Man kann daraus schlussfolgern, für wie wichtig die ArbeitgeberInnen den Sprachunterricht halten und welche Bedingungen sie bereit sind dafür zu gewährleisten. Die Tatsachen, dass die Mehrheit der ArbeitgeberInnen zulässt, dass der Sprachkurs innerhalb der Arbeitszeit stattfindet, und dass fast zwei Drittel der ArbeitgeberInnen die Kosten für den Sprachunterricht übernehmen, deuten darauf hin, dass der Sprachunterricht als wichtiger Bestandteil der Professionalisierung der Angestellten gesehen wird.

Was die Motivation der KursteilnehmerInnen anbelangt, ergab sich aus der Umfrage, dass sie unterschiedlich und auch sehr individuell geprägt ist. Daher wäre für die LektorInnen empfehlenswert, am Anfang jedes Sprachkurses eine kleine Umfrage oder eine Diskussionsrunde zu organisieren, wo die Lernmotivation der KursteilnehmerInnen thematisiert wird. Dabei kommen persönliche Wünsche und Prioritäten zum Vorschein, die die LektorInnen in ihrem Unterricht berücksichtigen sollten. Nicht zuletzt können solche Verfahren auch bei den KursteilnehmerInnen zu einer tieferen Bewusstmachung führen. Sie können sich dadurch ihrer eigenen Ziele und Wünsche bewusst werden und eigene Strategien vorschlagen, wie sie diese erreichen bzw. erfüllen verwirklichen.

Bezüglich der Methodik bzw. Didaktik des Fremdsprachenunterrichts in den Firmenkursen lassen sich auf Grund der Ergebnisse der durchgeführten Umfrage folgende Charakteristiken bzw. Spezifika formulieren: Die überwiegende Mehrheit der Firmenkurse wird von einem/einer tschechischen Lektor/in geleitet, was häufig auch der Wunsch der Befragten ist. Dieser Tatsache könnte eine eher konservative Denkweise sowohl der FirmenleiterInnen als auch der KursteilnehmerInnen zugrunde liegen. Man stellt sich unter einem/einer deutschen oder österreichischen Muttersprachler/in sehr oft jemanden vor, der kein Tschechisch spricht, was als Nachteil betrachtet wird. Hier ist zu betonen, dass die Sprache, die der/die Lektor/in

1 Die Autorin ist unter der E-Mail-Adresse pmareckova@ped.muni.cz erreichbar. 
beherrscht, nicht der entscheidende Faktor bezüglich der Qualität des Unterrichts ist, sondern seine/ihre methodisch-didaktische Kompetenz.

$\mathrm{Zu}$ den bevorzugten Tätigkeiten im Sprachunterricht zählen vor allem Konversations, Wortschatz- und Grammatikübungen. Was die Sozialformen betrifft, wird im Unterricht oft frontal sowie in Paaren oder individuell gearbeitet. Sehr wenig wird die Gruppenarbeit eingesetzt. Eine plausible Erklärung dafür könnte sein, dass die Anzahl der TeilnehmerInnen in diesen Sprachkursen viel zu niedrig ist, um Gruppen bilden zu können. Die Unterrichtsmaterialien werden meistens kombiniert und variiert, wobei ein Lehrwerk und kopierte Unterlagen zu den üblichsten Materialien zählen. Hingegen wird nur selten mit dem Internet gearbeitet. Der Grund dafür könnte sein, dass es technisch nicht möglich ist, während des Unterrichts im Internet zu arbeiten (keine PCs im Unterrichtsraum oder kein Internetzugang). Man sollte jedoch bedenken, dass das Internet auch im Rahmen der Hausaufgaben genutzt werden kann, was zweifelsohne aktivierend und zugleich motivierend für die KursteilnehmerInnen wäre.

Laut den Aussagen unserer RespondentInnen fühlen sich die meisten Befragten im Sprachunterricht durch nichts gestört und bewerten die Atmosphäre im Sprachkurs sowie die Kompetenz der LektorInnen positiv. Die Mehrheit der RespondentInnen gibt an, dass der Kurs ihre Erwartungen erfüllt bzw. erfüllt hat. Solche positiven Rückmeldungen können auf ihre wirkliche Zufriedenheit mit dem Sprachkurs hindeuten. Man sollte aber auch bedenken, dass einige KursteilnehmerInnen eher aus Passivität bzw. mangelndem Engagement dazu tendieren, alles weniger kritisch zu beurteilen.

Obwohl sich die Ergebnisse nicht verallgemeinern lassen, weil die Stichprobe nicht ausreichend groß war, ergaben sich im Laufe der Untersuchung weitere Themenbereiche, die detaillierter erforscht werden sollten (z. B. die instrinsische und extrinsische Motivation zum Fremdsprachenlernen bei Erwachsenen, die Binnendifferenzierung in Firmenkursen u. a.).

Zusammenfassend kann man sagen, dass der Sprachunterricht in Firmenkursen einen spezifischen Zugang verlangt. Nur schwerlich ließen sich zwei Firmenkurse finden, die nach dem gleichen Schema unterrichtet werden könnten. Jede Firma oder Institution hat, was den Fremdsprachenunterricht angeht, eigene Zielsetzungen, die in der Konzeption des Firmenkurses berücksichtigt werden sollten. Es gibt aber nicht nur langfristige, sondern auch kurzfristige Ziele, wie z. B. dienstliche Auslandsaufenthalte, auf die sich die Angestellten sprachlich vorbereiten sollen. Solche Firmenkurse sind stark an einem bestimmten Zweck orientiert und folgen dementsprechend einem komplett anderen Lehr- und Lernplan.

Jede Unterrichtseinheit, jede Lerngruppe und jeder Sprachkurs ist einzigartig. Diese Regel gilt im Lehrberuf allgemein, aber im Firmenunterricht gleich doppelt. 


\section{Literatur:}

GERR (2000): Europarat der EU. Gemeinsamer europäischer Referenzrahmen für Sprachen: lernen, lehren und beurteilen (online), 2000. Abgerufen am 10.9.2009 unter: http://www.goethe.de/referenzrahmen.

MAREČKOVÁ (2009): Marečková, Pavla. Deutschunterricht in Firmenkursen I, in: Brünner Hefte zu Deutsch als Fremdsprache, 2009, 2, Nr. 2, 48-64.

NEKULA (2004): Nekula, Marek. Deutsch als Europasprache aus tschechischer Sicht, in: Lohse, Ch. (Hg.): Die deutsche Sprache in der Europäischen Union. Rolle und Chancen aus rechts- und sprachwissenschaftlicher Sicht. Baden-Baden: Nomos Verlagsgesellschaft, 2004, 129-144. 